\title{
Novel Analytical Model of Stress Concentration around Broken Fibers of Unidirectional Composite Plate
}

\author{
Bachir Bouhamida', Abderrazek Merzoug ${ }^{1 *}$ \\ ${ }^{1}$ Laboratoire de Structures de Composites et Matériaux innovants, Faculté de Génie Mécanique, Université des Sciences et de la \\ Technologie d'Oran Mohamed-Boudiaf El Mnaouar, P. O. B. 1505, 31000 Bir El Djir, Oran, Algeria \\ * Corresponding author, e-mail: Abderrazek.merzoug@univ-usto.dz
}

Received: 30 March 2020, Accepted: 26 November 2020, Published online: 01 March 2021

\begin{abstract}
During the fiber fracture of unidirectional composite a distribution of stress around the neighbored fibers happens, this mechanism is called the local redistribution efforts. Referring to the "shear lag" method, the researches wanted to predict the stress concentration in the surrounding area of broken fibers as well as the longitudinal resistance of the unidirectional composite which presents a fiber breaking. The goal of this paper is to develop a new probabilistic model of unidirectional composite plate to calculate the stress concentration at the broken fibers and their neighboring fiber intact. The "shear lag" method has been generalized to see the broken fibers interference on the stress concentration factor variation of surrounding sound fibers.
\end{abstract}

Keywords

shear lag, broken fibers, stress concentration, unidirectional composite

\section{Introduction}

Actually, the composite materials are using in various industries due to their high mechanical performance. Therefore, the mathematics model, design and manufacturing process are necessary to assure a good interface fiber/matrix. The resistance of composite materials can be predicted not only by the resistance average of its constituents, but by the determination of the Stress Concentration Factor (SCF) around the broken fibers and their neighbored. The "shear lag" is the wide method used to modeling the interaction of fiber/matrix. Besides, it leads to predict the stress concentration in the surrounding area of broken fibers as well as the longitudinal resistance of unidirectional composite include broken fibers.

Rosen [1] and Zweben [2] are ones of the first creators of mathematic model to predict the polymer composite resistance. They determined the effective fiber length to estimate the optimum tensile resistance. Moreover, they made a shear model analysis of unidirectional composite materials, where neglected the effect of stress concentration on the neighbored fibers to that broken was taken. Phoenix et al. [3] were carried out a model to calculate the stress and life duration statistic of seven carbon fibers arranged under hexagonal shape reinforced epoxy resin. Landis et al. [4] were focused on the direct interaction of broken fiber and their neighbored using finite element methods of length composite fiber. Next, the last model was enhanced by Landis and McMeeking [5], where taken into account the axial slipping effect on the interface fiber/matrix and the effects of intact fibers position on the stiffness during the evaluation of stress concentration of around broken fiber. Case et al. [6] were performed a different technique to that previous, to study the behavior of unidirectional composite supporting a broken fibers. Spacing of fibers was the most important factor of this model to compare with reality of intact fibers neighbored. The succession of the fiber breaking has been modeled by means of the actualization method. Case and Reifsnider [7] treated the breaking in the center of several concentrically cylinders by adopting the standard elasticity hypothesis and by choosing the appropriate functions of each component. The same process has been applied with geometrical hypotheses in the case of fiber breakage in a unidirectional composite material. Foster [8] suggested a direct numerical simulation and an analytical model to predict the resistance of composite in tensile and flexural. Using a square shaped structure of surrounding fibers, the researcher determined the resistance of Ti-6Al-4V metallic matrix reinforced by SiC. They also showed that fiber breaking takes place in a random direction 
when the load reaches the resistance limit of the composite. Thus, the statistical accumulation of this breaking leads to the complete damaging of the composite. Gao and Reifsnider [9] were suggested the using of Batdorf probabilistic model for the repartition loading analysis to predict the tensile resistance. Based on shear lag hypotheses, they studied the fracture of composite due to the interface fiber/ matrix. The shear parameter to describe the functionality of elastic composite was taken as well as the thoroughly plastic debonding of matrix. Goda [10] proposed a probabilities model of resistance that is based on the process of the Markov chain for the unidirectional fibers composite in a hexagonal shape. This model takes into account only one fibers group of hexagonal structure. The damaging of this group changes proportionally with the load. Costa et al. [11] present a model of composites progressing damage that contain initially broken fibers. The distribution of Weibull parameters of the fibers resistance and the mechanical properties to the fiber-matrix interface has permitted to determine the loss rigidity of the composite. Rouhi and Rais-Rohani [12], have studied the elastic properties of a random carbon nanofiber (CNF) reinforced a vinylester matrix under buckling loading. They introduced the micromechanical approaches on the mathematical modeling. Besides, they considered the inter-phase mechanical properties to vary in a manner similar to functionally graded materials. As results, their model led to calculate the optimum design values for the thickness and radius of a hybrid composite cylinder and quantify the required value for each design variable in terms of a prescribed maximum probability of failure in a buckling problem.

In this work, an attempt to calculate the stress concentration factor in unidirectional composite plate, where it contains several broken fibers that are dispersed or grouped. The "shear lag" method has been generalized to see the broken fibers interference on the stress concentration factor variation of surrounding fibers. A comparative study was made to Bandorawalla [13] the results have checked and proved thanks to the comparison made between the different common approaches.

2 The "shear lag" method of a unidirectional composite A plate of unidirectional composite loaded in the fibers direction has been modeled. When all fibers are intact, the composite material presents a uniform aspect of axial united stresses. But if a fiber broke, a micro-mechanical redistribution occurs near to the broken fibers. This stress is transmitted to the neighboring fibers through the matrix.
The NNLS (Nearest Neighbor Load Sharing) and HVDLS (Hedgepeth and Van Dyke [14], and Load Sharing) methods have been adopted to determine the stress concentration factor of a unidirectional composite loaded with a uniform tensile loading.

Fig. 1 shows a hexagonal arrangement with regular and irregular spacing between fibers.

According to Bandorawalla [13], the distance between the fibers centers are represented as follows:

$s=\sqrt{\frac{\pi}{V_{f} \sin 60^{\circ}} r_{f}}$.

To be in accordance with the description given in [13], the broken fiber number $(i, j)$ "37" and the neighboring fibers have been numbered from 1 to $N \times M$ (see Fig. 1). It is important to point out that the hexagonal shape of fibers is essential to calculate the general influencing strengths of the broken and those of neighboring intact fibers. It is illustrated by Eq. (2):

$\{Q\}=\left[\begin{array}{c}q_{1,1}(x) \\ q_{1,2}(x) \\ \vdots \\ q_{n, m}(x)\end{array}\right]$.

For the displacement vector of the fibers that constitute the hexagonal plate $u_{1,1}(x), u_{1,2}(x) \ldots . u_{n, m}(x)$ :

$\{U\}=\left[\begin{array}{c}u_{1,1}(x) \\ u_{1,2}(x) \\ \vdots \\ u_{n, m}(x)\end{array}\right]$,

when referring to the "shear lag" hypothesis, we can get:

$\{Q\}=E_{f} \frac{d}{d x}\{U\}$

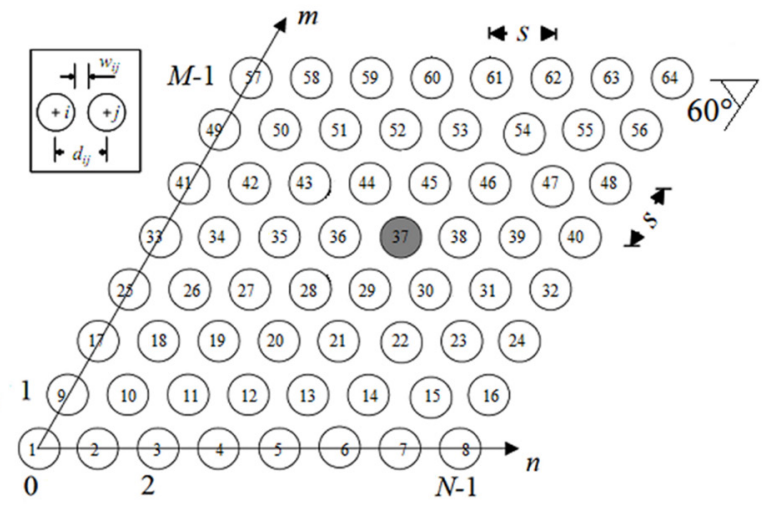

Fig. 1 Arrangement with hexagonal fiber numbered. 
Using the demonstration in Fig. 1, the space between the $i$ and $j$ fiber center is expressed as follows: $d_{i j}$, with $w_{i j}=d_{i j}-2.0 r_{f}$. The "shear lag" hypothesis is used for the $N \times M$ fibers plate. The equations system for displacement is:

$$
\frac{d^{2}}{d x^{2}}\{U\}+[A]\{U\}=0 .
$$

[A] is a square matrix $[N \times M, N \times M]$ of which elements are expressed with:

$$
\begin{aligned}
& A(n i, n i)=\sum_{j=1}^{6} \frac{-C}{w_{i j}}:(n i=1 \ldots N \times M, i=1 \ldots N) \\
& A(n i, n j)=\frac{C}{w_{i j}}:(n i, n j=1 \ldots N \times M) \\
& C=\frac{h G_{m}}{A_{f} E_{f}} .
\end{aligned}
$$

$h$ is the matrix-width expressed as $h=(\pi r f) / 3$. Limit conditions to calculate the influence functions are:

$u_{n j}(0)=1$,

$u_{n i}(0)=1 \quad: \quad n i=1 \ldots N \times M \quad(n j \neq n i)$

and

$\left.\frac{d}{d x}\{U\}\right|_{x=\infty}=0$.

We considerate an irregular arrangement fibers that has a varying diameter (Fig. 2), the displacement of the fiber $(i, j)$ on the $x$ direction is expressed as $U_{i, j}(x)$ and Eq. (5) is:

$$
\frac{d^{2} U_{i, j}}{d x^{2}}+C\left(\begin{array}{l}
\frac{u_{i-1, j}}{w_{i-1, j}}+\frac{u_{i-1, j+1}}{w_{i-1, j+1}}+\frac{u_{i, j+1}}{w_{i, j+1}}+\frac{u_{i+1, j}}{w_{i+1, j}} \\
+\frac{u_{i+1, j-1}}{w_{i+1, j-1}}+\frac{u_{i, j-1}}{w_{i, j-1}}-6 \frac{u_{i, j}}{w_{i, j}}
\end{array}\right)=0 .
$$

\section{Developed numerical model}

The differential equation (Eq. (11)) can be calculated with the finite differences method with simple boundaries; the general displacement of fibers is equal zero, but in the case of broken fiber the displacement will be equal to one. Fig. 3 shows the discretization based on the finite differences model and the adding of fictive knots on the four sides $(i=0, j=0, i=n+1$ and $j=m+1)$.

Thus, the calculation of each fiber displacement can be represented by Eq. (12):

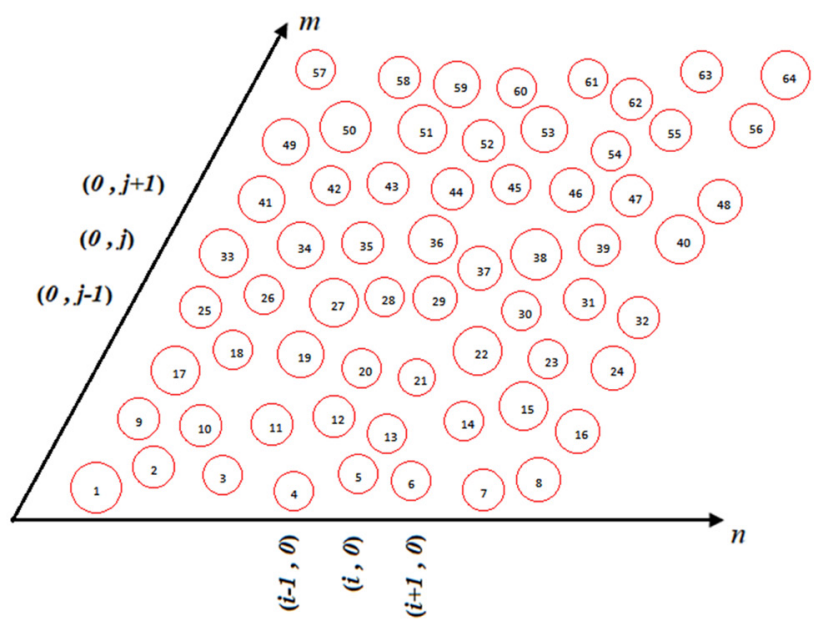

Fig. 2 Irregular hexagonal structure with varying diameter and fibers numbering.

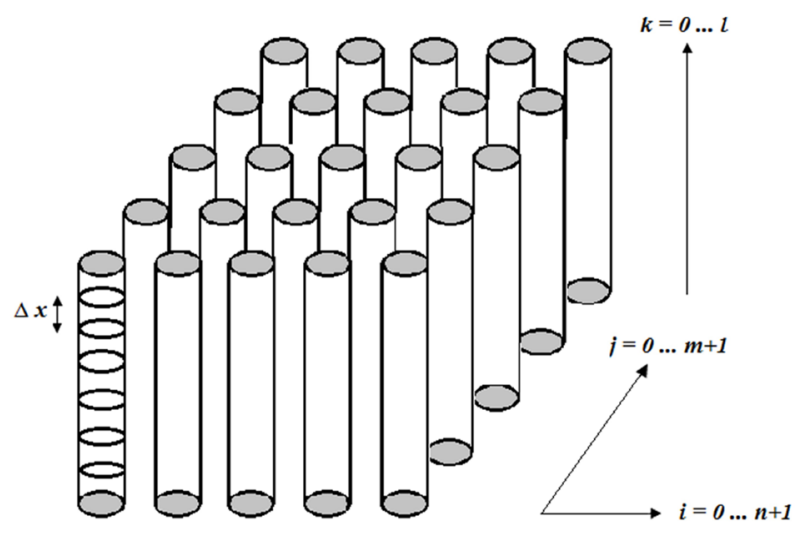

Fig. 3 Discretization as the finite difference model.

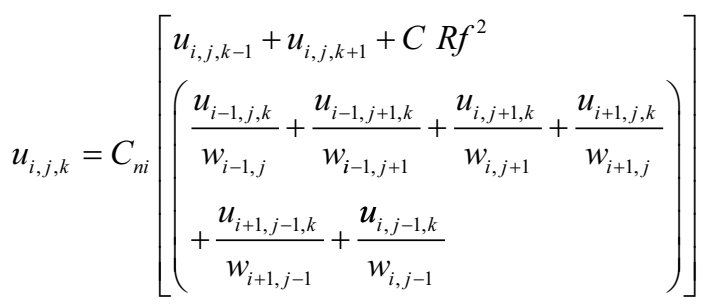

with $C_{n i}=\frac{1}{\left[2+C R f^{2} A(n i, n i)\right]} \quad: \quad n i=1 \ldots N \times M$.

To calculate the stress concentration factor, we must calculate the derivatives of displacements along with the three following formulae: the front, center and back:

$$
\begin{aligned}
& d u_{i, j, k}=E f\left(\frac{u_{i, j, k+1}-u_{i, j, k}}{\Delta x}\right), d u_{i, j, k} \\
& =E f\left(\frac{u_{i, j, k+1}-u_{i, j, k-1}}{2 \Delta x}\right) o u d u_{i, j, k} \\
& =E f\left(\frac{u_{i, j, k}-u_{i, j, k-1}}{\Delta x}\right) .
\end{aligned}
$$


The stress concentration factor can be calculated for all fibers using:

$C f_{i, j, k}=1-\frac{d u_{i, j, k}}{d u_{i, j, 1}}$.

\section{Validation}

\subsection{One broken fiber}

In order to evaluate the efficiency of the model proposed, a validation was achieved with other analytical models existed. The first validation has been carried out through Bandorawalla [13], which compared the HVDLS and the NNLS models that permit the local distribution loading for one broken fiber in the equidistant case as shown in Fig. 4.

The NNLS approach supposes that the stress concentration factor generated by the broken fiber and spread on the nearest neighboring fibers only. In the case of hexagonal arrangement, the nearest fibers are six in number; this is why we can distribute the broken fiber SCF on the six fibers accurately.

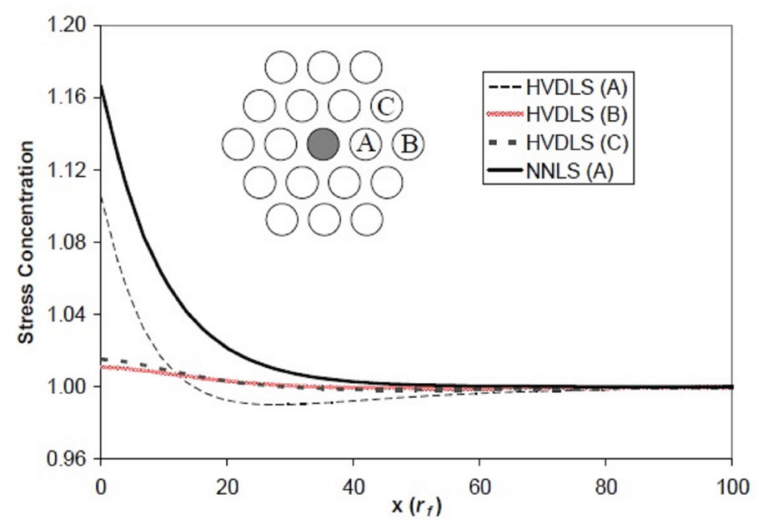

(a)

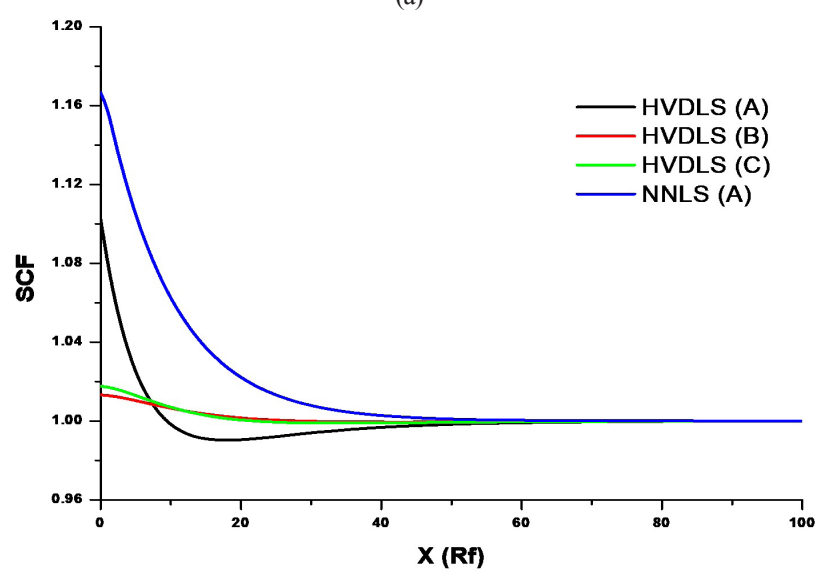

(b)

Fig. 4 Comparison between NNLS and HVDLS of neighboring fibers; (a) Bandorawalla result [13]; (b) Present model results.
In contrast, the HVDLS approach supposes that the stress concentration factor generated by the broken fiber is not only spread on the nearest neighboring fibers but beyond these fibers also. Fig. 5 shows the distribution of the SCF in the plate shown in Fig. 1. The distribution occurs in the cell that contains the broken fiber and the SCF is well spread on the whole plate. The experiment has been proved adequate through $10^{5}$ necessary iterations.

A PPS graphite-composite has been used in our general applications. The disturbance distance from the break on the length is $x_{p}$ till $100 \times r_{f}$. Table 1 presents both geometrical and mechanical characteristics of PPS-graphite unidirectional composite.

Fig. 5 presents a plate in which the SCF is not symmetrically done around the broken fiber because of the broken fiber position; this latter isn't in right center. To prove a real symmetry of SCF spreading around the broken fiber, we have to choose an odd number of strata. For example, the number 9 illustrates the idea in Fig. 6.

Fig. 7, present our results validate with Hedgepeth and Van Dyke [14] method, where the hexagonal arrangement of fiber contain a broken fiber. Fig. 7 shows the stress concentration factor variation that based on the broken fiber

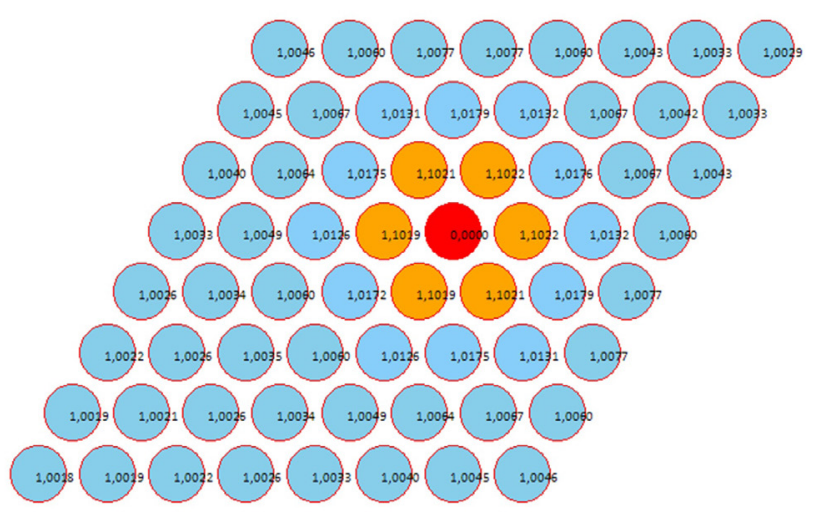

Fig. 5 SCF distribution based on our results.

Table 1 Geometrical and mechanical characteristics of PPS-graphite unidirectional composite

\begin{tabular}{lc}
\hline Young's modulus of the fiber $E_{f}$ & $234.4 \mathrm{Gpa}$ \\
\hline Matrix Young's modulus $E_{m}$ & $4.4 \mathrm{Gpa}$ \\
Fiber reference resistor $\sigma_{0}$ & $3.17 \mathrm{Gpa}$ \\
Shear stress $\tau_{0}$ & $25.8 \mathrm{Mpa}$ \\
Poisson's ratio of the matrix $v$ & 0.43 \\
Volume fraction of fibers $V_{f}$ & 0.6 \\
Shear parameter $\eta$ & 1.0 \\
Radius of the fiber $r_{f}$ & $3.5 \mu \mathrm{m}$ \\
Disturbance distance $x_{p}$ & $100 \times r_{f}$ \\
\hline
\end{tabular}




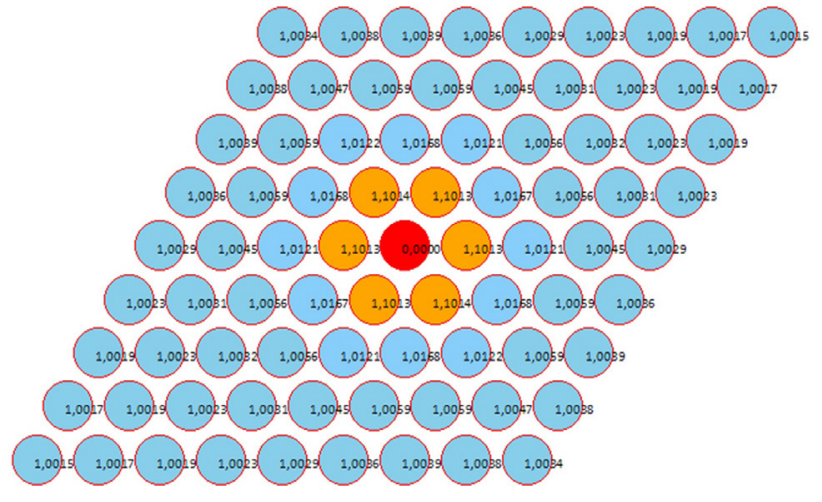

Fig. 6 Symmetry of the distribution of SCF.

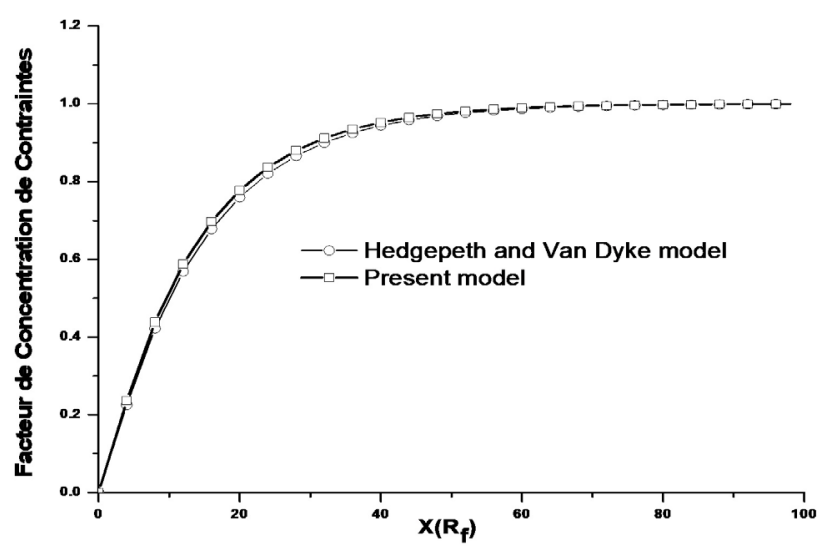

Fig. 7 Comparison between HVDLS and NNLS for the broken fiber.

length. The results of our model are in perfect harmony with those of the HVDLS. The stress concentration factor is zero at the $x=0$ position. However, the SCF become 1 for the important fiber length. On one hand, Fig. 4 shows the change of the stress concentration factor in relation with the neighboring fibers length. We can notice a difference between the NNLS and the HVDLS models for fiber (A), especially for $x=0$. Both curves convergent to the same value $\mathrm{SCF}=1.0$ when towards more important lengths. On the other hand, the HVDLS brings a less stress concentration factor around the sound neighboring fiber, i.e. 1.10 for each nearest neighboring fiber. Consequently, the remaining stress concentration is spread over the border of the nearest neighboring fibers as it is show in Fig. 5.

\subsection{Two broken adjacent fiber}

According to Phoenix et al. [3] (quasi-LLS), the second fiber break gives birth to equal load distribution over the six neighboring fibers. SCF that equals 1.166, as in Fig. 8 (a). Moreover, $1 / 15$ is transmitted to the five following fibers (A, B, F, G, H). This doesn't coincide with the LLS rule that is developed by Goda [10] Fig. 8 (b). Recently, Swolfs et al. [15] have entered a correcting coefficient to get a strength balance as Fig. 8 (c). (a)

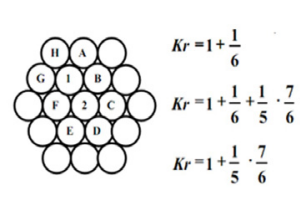

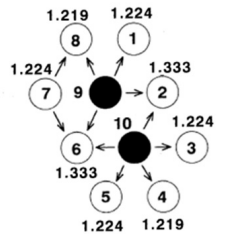

(b)

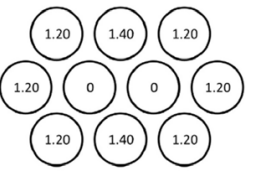

(c)
Fig. 8 Stress concentration in the case of two broken fibers according to (a) Phoenix model [3]; (b) Goda model [10]; and (c) Swolfs model [15].

The results determined by our model was in perfect correlation to Swolfs model, known as we did not used a correction coefficient to reach the equilibrant force. This has been reached by repeating the same model iteration at least $10^{5}$, and by taking very small discretization step $\Delta x$ as in Fig. 9.

\section{Applications \\ 5.1 One broken fiber}

The fibers in a hexagonal unidirectional can never be placed in equidistant, this is why we take into account the in-between fibers distance as well as the varying fibers diameters. When we compare both nine strata plates and we maintain the constant diameter, we can see that plate (Fig. 10 (a)) shows in Fig. 10 at random given distance whereas plate (Fig. 10 (b)) presents at random given diameter and distance. When a fiber breaks in the middle (41), a strength distribution spreads on the other round fibers.

The broken fiber concentration distribution happens according to two main criteria. These are the distance that separates the broken fiber from the around ones and the fiber diameter Bouhamida et al. [16]. Fig. 11 shows both distributions on the intact fibers. In Fig. 11 (a) nearest fibers of the broken one present a more important SCF than the one of fiber 50, fiber 42 and fiber 49. But fiber 40 that is the remotest of the 6 fibers, takes the most little SCF of the group. Fig. 11 (b) shows that the second criterion is taken into consideration to differentiate the distribution because the 33, 40 and 49 are located at the same distance.

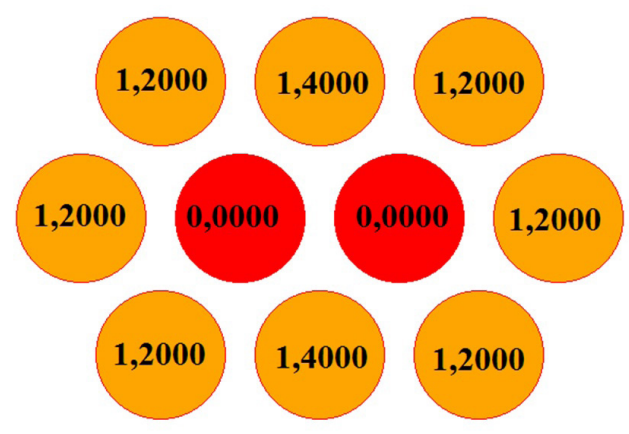

Fig. 9 Stress concentration in the case of two broken fibers according to our results. 


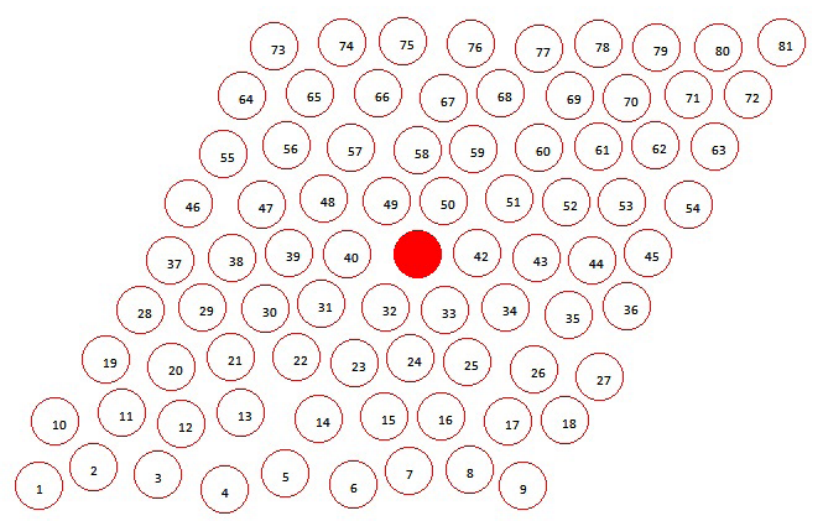

(a)

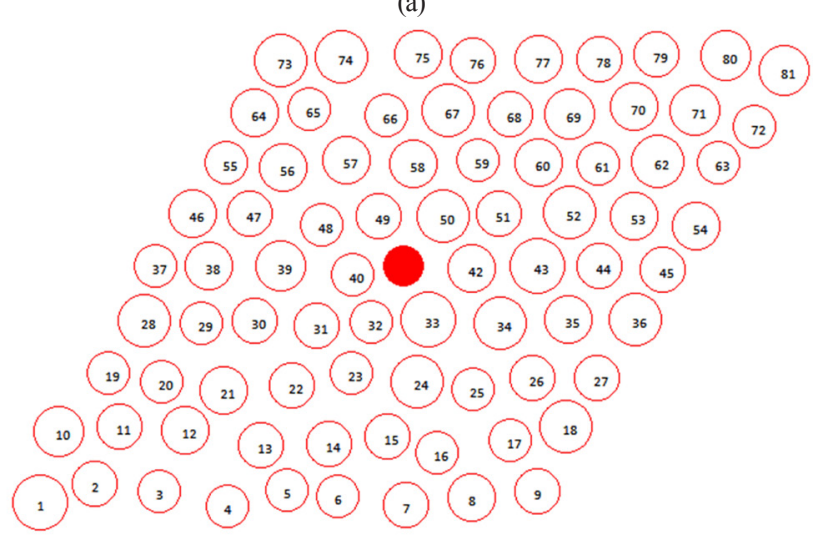

(b)

Fig. 10 Plate of nine layers with the middle fiber is broken; (a) Distance random and constant diameter; (b) Distance random and variable diameter.

Fiber 40 has a lowest diameter than that of fiber 49 and the most important diameter is the fiber 33 , so the fiber 40 takes the highest SCF than that fiber 49 and 33.

\subsection{Two broken fibers}

Fig. (12) shows that the fiber 40 is the most solicited. Both broken fibers require a third criterion that differentiates the SCF distribution over the around fibers. If the around fiber is adjacent to both broken fibers, it takes more SCF. Fig. 12 presents the SCF of the neighbored fibers of fiber 40 and fiber 41 . The example of fibers adjacent to the broken and fiber 32 and fiber 49 having mostly the same diameter reveals that fiber 32 is most solicited because of its location near the broken fiber.

\subsection{Three broken fibers}

The program has permitted to draw a great deal of random pictures that show the fiber qualities and the diameters variation. The number of broken fibers permits to differentiate the SCF distribution. The tow be-broken fiber is fiber 32. Fig. 13 illustrates the SCF distribution of the 40, 41 and 31 broken fibers.

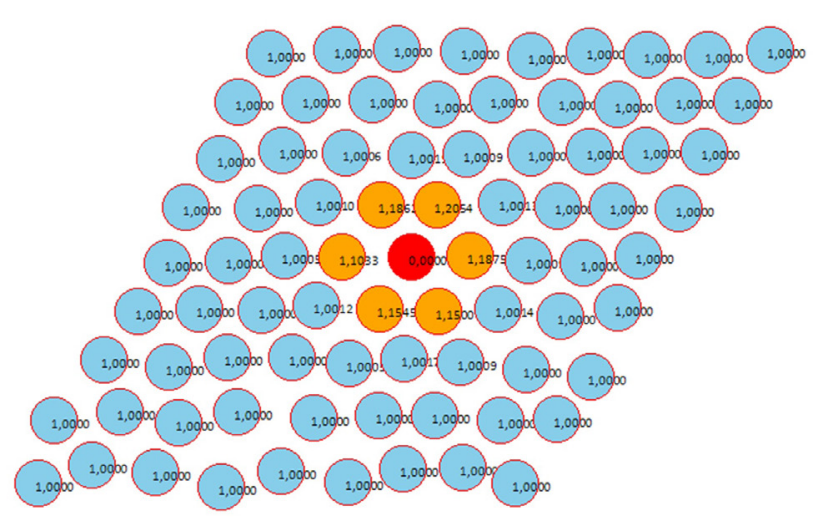

(a)

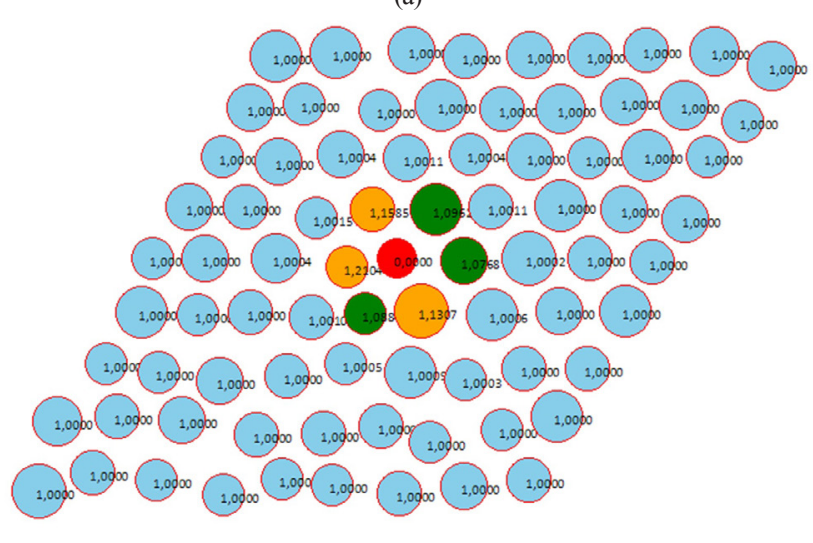

(b)

Fig. 11 SCF distribution around a broken fiber; (a) Random distance and constant diameter; (b) Random distance and variable diameter.

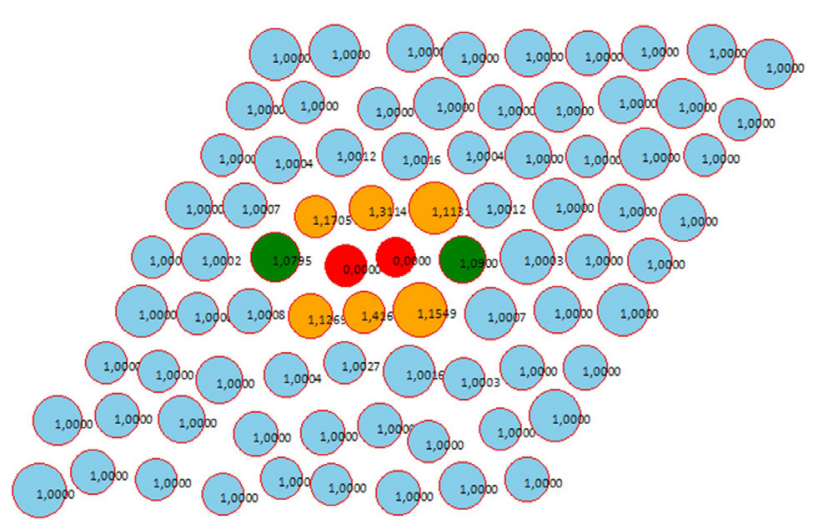

Fig. 12 CFS distribution around two broken fibers.

\section{Reliability}

\subsection{Determinism case}

To determine the results easily and accurately, we can't break each given fiber continuously but we can go for Monte Carlo method. Firstly, experiments have been carried out with one broken fiber in the middle taking into account deterministic view. The SCF distribution scope is less important: the stress concentration variation goes from 1.1002 to 1.1057 . The histogram shows a display gap and a very small variation coefficient in Fig. 14. 


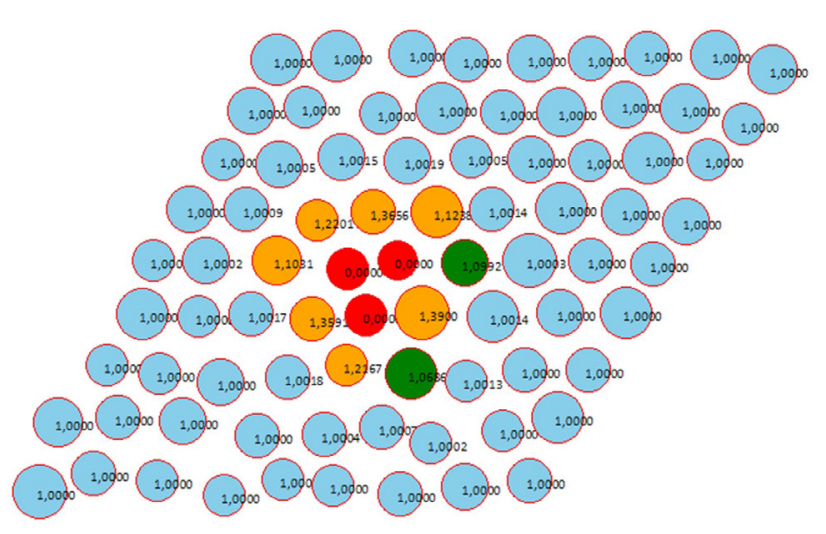

Fig. 13 Distribution of the SCF on three broken fibers.

\subsection{The same net random case}

Secondly, experiments have been led with one broken fiber in the middle, keeping the same netting but in a random way. The SCF from distribution scope is limited. The stress concentration variation is not important: it goes 1.18 to 1.20 . The distribution here doesn't change since the fibers and their diameters are kept with the same random qualities which are influential criteria over the SCF condition: the distance between the second fiber and the broken one and the fiber's diameter (Fig. 15).

\subsection{Different netting random case}

Thirdly, simulated experiments have been conducted with a broken fiber; the fiber in the middle by having a new netting each time. The SCF distribution scope spreads from 1.14 to 1.30 . So the difference with the above examples is clear. The distribution has significantly grown. The histogram in Fig. 16 presents the new netting that includes different distances between the fibers and their varying diameters.
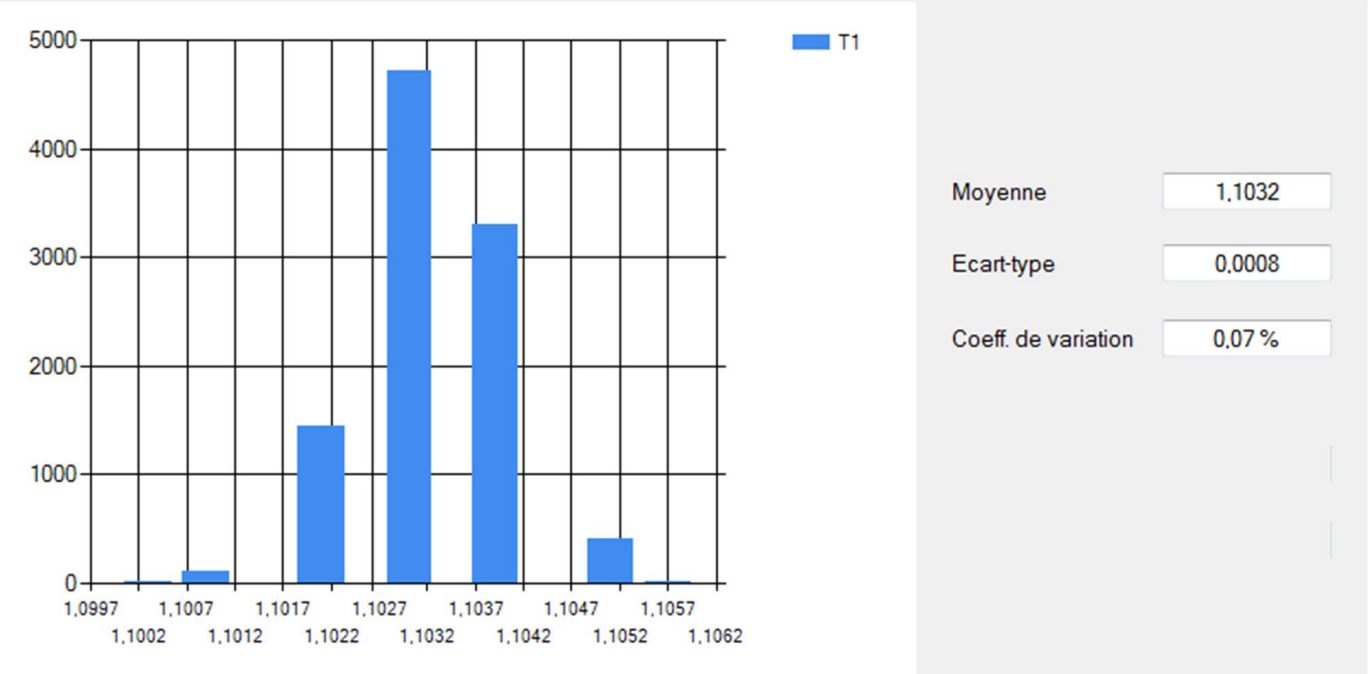

Fig. 14 SCF distribution in the deterministic case with one broken fiber.
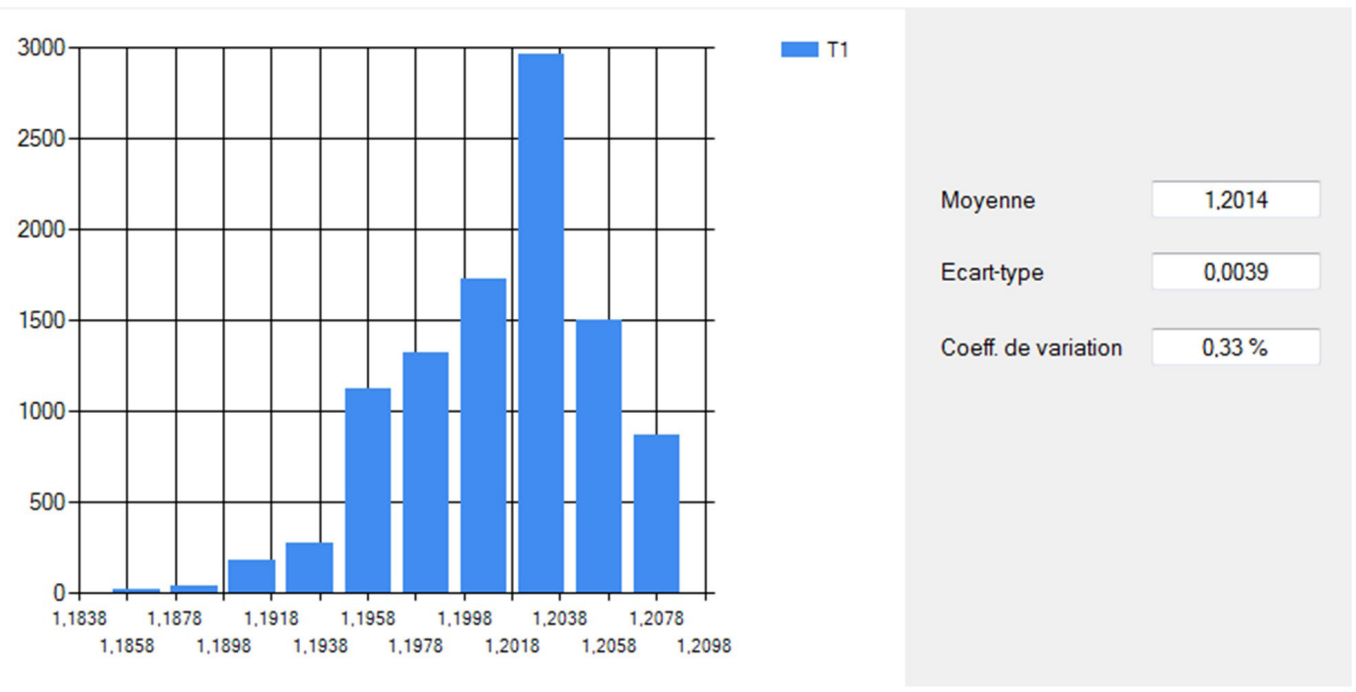

Fig. 15 SCF distribution in the case of the same random arrangement with one broken fiber. 


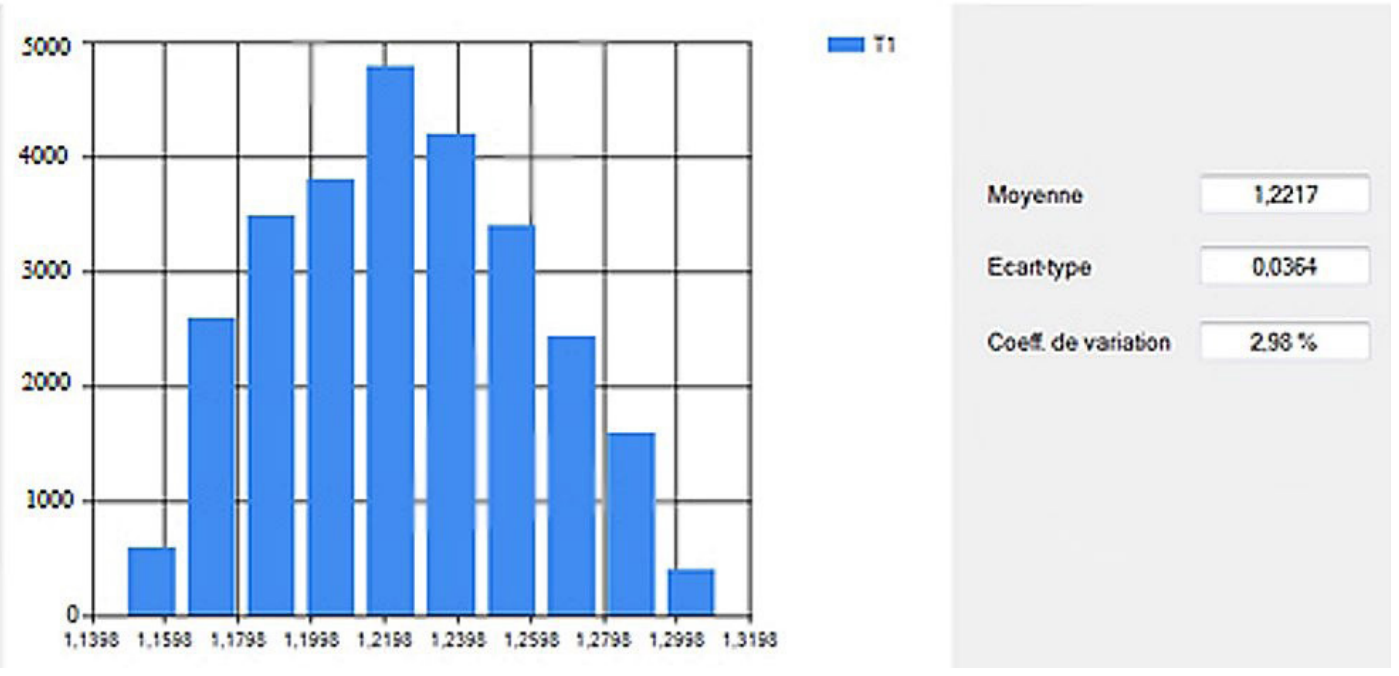

Fig. 16 SCF distribution in the case of a new mesh for each draw.

\subsection{Spreading}

In this case, there are three fibers. After the central fiber breaking, the crack has spread from one fiber to another. This crack spreading gives from the broken fiber to the nearest round one. Fig. 17 displays the SCF distribution of which scope is very important and goes over 1.5.

If we carry on the same experiment: the crack spreading over more than three fibers and using 2.0 SCF limit for a fiber, we can notice that fiber 16 has gone the limits after the breaking of several fibers (9) and it shows an SFC of 2.1803 see Fig. 18.

\section{Conclusion}

The aim of the present study is to determine the SCF distribution of one or several broken fibers over the around fibers of a nine strata plate using a new model. When only one fiber is broken, the SCF is distributed on the whole plate in a digressive way and a maximum SCF quantity is taken back by the fibers, which are adjacent to the broken fiber. Two criteria can greatly influence significantly the stress concentration distribution such as the distance between the intact fiber and the broken fiber and the fiber's diameter. For two broken fibers and more, a third criterion influence the distribution of SCF is the number of $n$ broken fibers. The SCF of intact fiber increases considerably if it is adjacent to several broken fibers.

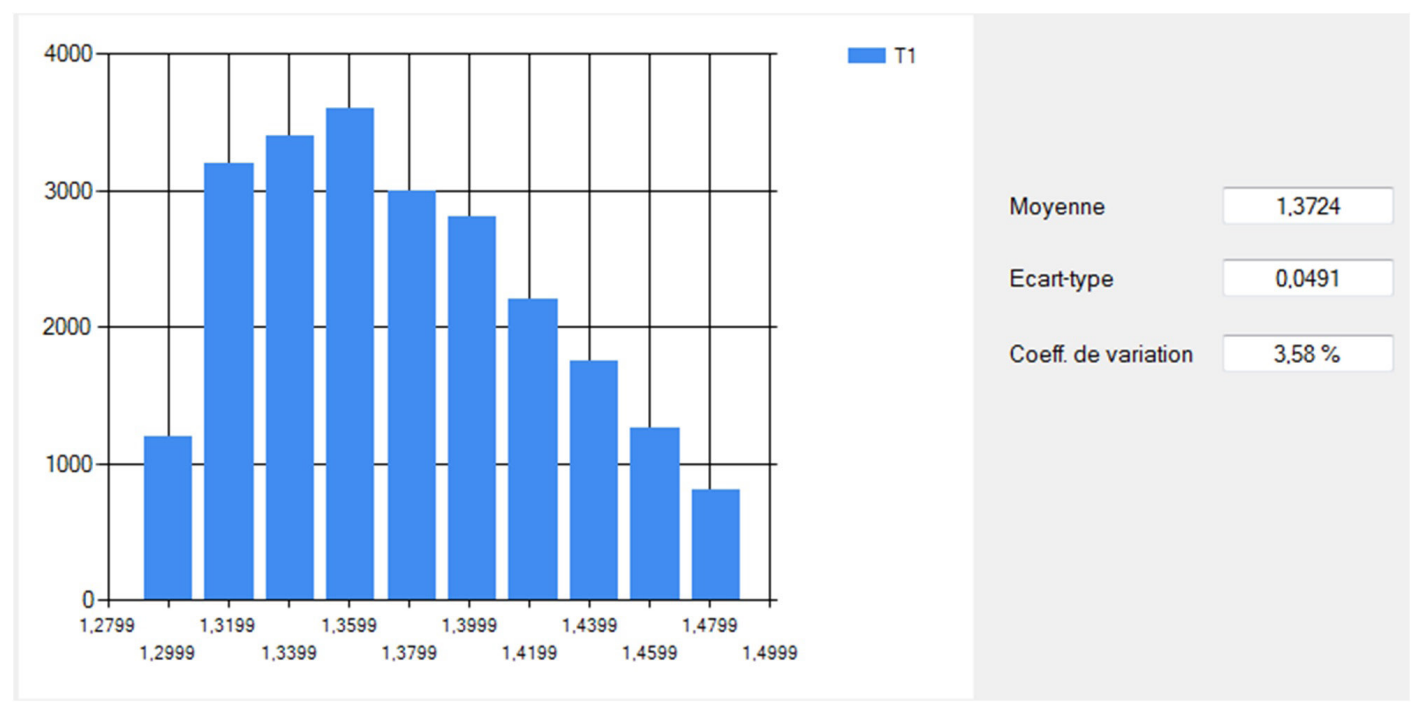

Fig. 17 SCF distribution in the case of crack propagation to three fibers. 


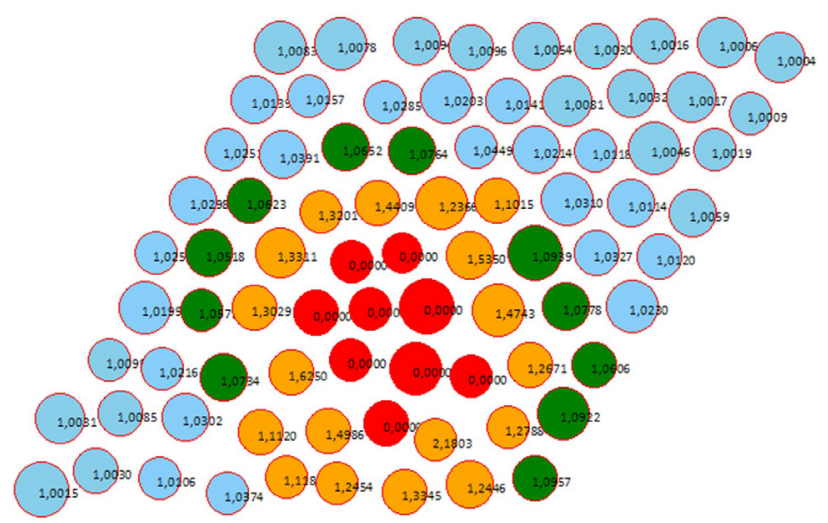

Fig. 18 The SCF around nine broken fibers.

\section{References}

[1] Rosen, B. W. "Tensile failure of fibrous composites", American Institute of Aeronautics and Astronautics, 2(11), pp. 1985-1991, 1964.

https://doi.org/10.2514/3.2699

[2] Zweben, C. "Tensile failure of fiber composites", American Institute of Aeronautics and Astronautics, 6(12), pp. 2325-2331, 1968. https://doi.org/10.2514/3.4990

[3] Phoenix, S. L., Schwartz, P., Robinson, H. H. "Statistics for the strength and lifetime in creep-rupture of model carbon/ epoxy composites", Composites Science and Technology, 32(2), pp. 81-120, 1988.

https://doi.org/10.1016/0266-3538(88)90001-2

[4] Landis, C. M., McGlockton, M. A., McMeeking, R. M. "An Improved Shear Lag Model for Broken Fibers in Composite Materials", Journal of Composite Materials, 33(7), pp. 667-680, 1999. https://doi.org/10.1177/002199839903300704

[5] Landis C. M., McMeeking R. M. "Stress concentrations in composites with interface sliding, matrix stiffness and uneven fiber spacing using shear lag theory", International Journal of Solids and Structures, 36(28), pp. 4333-4361, 1999. https://doi.org/10.1016/S0020-7683(98)00193-0

[6] Case S. W., Carman, G. P., Lesko, J. J., Fajardo, A. B., Reifsnider, K. L. "Fiber Fracture in Unidirectional Composites", Journal of Composite Materials, 29(2), pp. 208-228, 1995. https://doi.org/10.1177/002199839502900205

[7] Case, S. W., Reifsnider, K. L. "Micromechanical analysis of fiber fracture in unidirectional composite materials", International Journal of Solids and Structures, 33(26), pp. 3795-3812, 1996. https://doi.org/10.1016/0020-7683(95)00214-6

[8] Foster, G. C. "Tensile and Flexure Strength of Unidirectional Fiber-Reinforced Composites: Direct Numerical Simulations and Analytic Models", MSc Thesis, Virginia Polytechnic Institute and State University, 1998. [online] Available at: https://vtechworks. lib.vt.edu/handle/10919/36688 [Accessed: 15 October 2019]

[9] Gao, Z., Reifsnider, K. "Micromechanics of Tensile Strength in Composite Systems", In: Stinchcomb, W., Ashbaugh, N. (eds.) Composite Materials: Fatigue and Fracture, ASTM International, West Conshohocken, PA, USA, pp. 453-470, 1993. https://doi.org/10.1520/STP24745S
[10] Goda, K. "A strength reliability model by Markov process of unidirectional composites with fibers placed in hexagonal arrays", International Journal of Solids and Structures, 40(24), pp. 6813-6837, 2003. https://doi.org/10.1016/j.ijsolstr.2003.07.003

[11] Costa, J., Turon, A., Trias, D., Blanco, N., Mayugo, J. A. "A progressive damage model for unidirectional fibre-reinforced composites based on fibre fragmentation. Part II: Stiffness reduction in environment sensitive fibres under fatigue", Composites Science and Technology, 65(14), pp. 2269-2275, 2005. https://doi.org/10.1016/j.compscitech.2005.05.011

[12] Rouhi, M., Rais-Rohani, M. "Modeling and probabilistic design optimization of a nanofiber-enhanced composite cylinder for buckling", Composite Structures, 95, pp. 346-353, 2013. https://doi.org/10.1016/j.compstruct.2012.05.035

[13] Bandorawalla, T. J. "Micromechanics-Based Strength and Lifetime Prediction of Polymer Composites", PhD Dissertation, Virginia Polytechnic Institute and State University, 2002. [online] Available at: http://scholar.lib.vt.edu/theses/available/etd-03172002-151608/ unrestricted/tozeretd.pdf [Accessed: 09 September 2019]

[14] Hedgepeth, J. M., Van Dyke, P. "Local Stress Concentrations in Imperfect Filamentary Composite Materials", Journal of Composite Materials, 1(3), pp. 294-309, 1967. https://doi.org/10.1177/002199836700100305

[15] Swolfs, Y., McMeeking, R. M., Verpoest, I., Gorbatikh, L. "The effect of fibre dispersion on initial failure strain and cluster development in unidirectional carbon/glass hybrid composites", Composites Part A: Applied Science and Manufacturing, 69, pp. 279-287, 2015.

https://doi.org/10.1016/j.compositesa.2014.12.001

[16] Bouhamida, B., Sereir Z., Chateauneuf, A. "Uncertainties of stress intensification near broken fibers of unidirectional composite with random fiber spacing", Theoretical and Applied Fracture Mechanics, 65, pp. 1-7, 2013.

https://doi.org/10.1016/j.tafmec.2013.05.001 